\title{
Biquadratic Spline Approximations
}

\author{
By \\ Manabu SAKAI* and Riaz A. USMANI**
}

\begin{abstract}
We shall consider two kinds of spline approximations over a uniform mesh. The first of them is mid-point spline interpolation and the second is histospline approximation. Our methods don't need any knowledge of derivative values, that is, end conditions are homogeneous ones dealing with function values. The linear systems for computing approximations may be conveniently and rapidly solved using the simple algorithm. Some numerical results are given which closely correspond with the prediction of the theory.
\end{abstract}

\section{$\S 1$. Introduction}

This paper is concerned with biquadratic spline approximations of certain smooth functions $f(x, y)$ in $\Omega=[0,1] \times[0,1]$. Let $n$ and $r$ be integers such that $n \geq r \geq 0(n \geq 0)$. By making use of $B-$ spline $Q_{3}(x)=\frac{1}{2} \sum_{i=0}^{3}(-1)^{i}\left(\begin{array}{c}3 \\ i\end{array}\right)(x-i)_{+}^{2}$, we take the following spline function $s(x, y)$ of the form

$$
s(x, y)=\sum_{i, j=-2}^{n-1} \alpha_{i, j} Q_{3}(x / h-i) Q_{3}(y / h-j), n h=1
$$

with $(n+2)^{2}$ undetermined coefficients $\alpha_{i, j}, i, j=-2(1) n-1$. It is known that

(i) in each region $\left[x_{i}, x_{i+1}\right] \times\left[y_{j}, y_{j+1}\right]\left(x_{i}=i h, y_{j}=j h, i, j=0(1)\right.$ $n-1) s$ coincides with an algebraic polynomial of degree 2 or less with respect to $x$ and $y$, respectively, (ii) $s \in C^{1}(\Omega)$.

First we consider the mid-point interpolation problem :

$$
s_{i+\frac{1}{2}, j+\frac{1}{2}}=f_{i+\frac{1}{2}, j+\frac{1}{2}}, i, j=0(1) n-1
$$

Communicated by S. Hitotsumatsu, September 13, 1982. Revised July 11, 1983.

* Department of Mathematics, Faculty of Science, Kagoshima University, Kagoshima 890, Japan.

** Department of Applied Mathematics, University of Manitoba, Winnipeg, Manitoba, Canada R3T 2N2 
where

$$
s_{i+\frac{1}{2}, j+\frac{1}{2}}=s\left(x_{i+\frac{1}{2}}, y_{j+\frac{1}{2}}\right)=s\left(\left(i+\frac{1}{2}\right) h,\left(j+\frac{1}{2}\right) h\right) .
$$

With the help of the consistency relation (Lemma 2.2), the values $s_{i, j}=s\left(x_{i}, y_{j}\right), i, j=0(1) n$ may be determined by interpolation condition (1.2) and $4 n$ appropriate additional conditions. Here we take these conditions to be boundary ones of the form :

$$
\left\{\begin{array}{c}
\Delta_{1}^{r}\left(s_{0, j+1}+6 s_{0, j}+s_{0, j-1}\right)=\nabla_{1}^{r}\left(s_{n, j+1}+6 s_{n, j}+s_{n, j-1}\right)=0 \\
j=1(1) n-1 \\
\Delta_{2}^{r}\left(s_{i+1,0}+6 s_{i, 0}+s_{i-1,0}\right)=\nabla_{2}^{r}\left(s_{i+1, n}+6 s_{i, n}+s_{i-1, n}\right)=0 \\
i=1(1) n-1, \\
\left(\Delta_{1} \Delta_{2}\right)^{r} s_{0,0}=\left(\nabla_{1} \Delta_{2}\right)^{r} s_{n, 0}=\left(\Delta_{1} \nabla_{2}\right)^{r} s_{0, n}=\left(\nabla_{1} \nabla_{2}\right)^{r} s_{n, n}=0
\end{array}\right.
$$

where $r=3,4$ and $\Delta_{1}, \Delta_{2}\left(\nabla_{1}, \nabla_{2}\right)$ are forward (backward) difference operators with respect to $x, y$, respectively. In practical computation, on using Lemmas 2.2-2.4 we have a system of linear equations with $s_{i, j}$ whose coefficient matrix is block tridiagonal. This system may be conveniently and rapidly solved using the sweep out method. The restriction $r=3,4$ is not incisive. Because the constant terms of the obtained system contain very large numbers in magnitude for $r \geq 5$. Since $s$ depends upon $(\mathrm{n}+2)^{2}$ parameters and conditions $(1.2)-(1.3)$ give us $n^{2}+4 n$ equations toward the determination of $s$, four additional conditions are required. On referring Figure 1, let us impose only one condition on each linear segment of the boundary :

$$
\Delta_{1}^{r} s_{\frac{1}{2}, 0}=\Delta_{2}^{r} s_{n, \frac{1}{2}}=\nabla_{1}^{r} s_{n-\frac{1}{2}, n}=\nabla_{2}^{r} s_{0, n-\frac{1}{2}}=0 .
$$

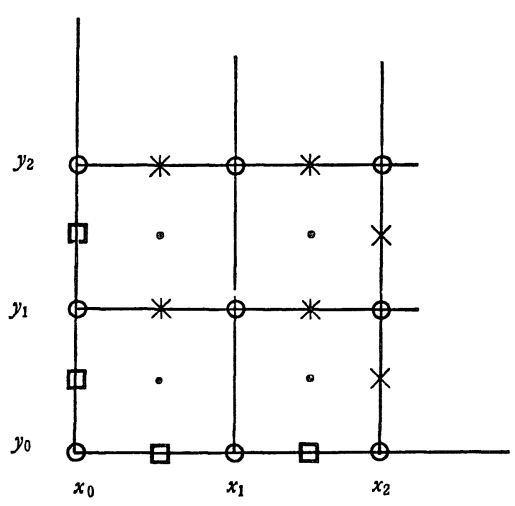

Figure 1

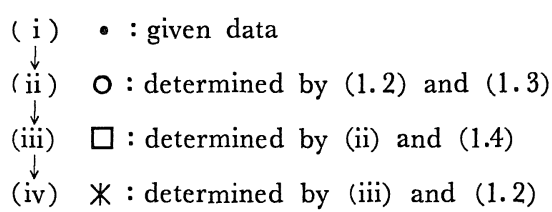


Since $s(0, y)$ is a quadratic spline, by means of a consistency relation at mesh and mid points we have

$$
\frac{1}{2}\left(s_{0, i+\frac{1}{2}}+s_{0, i-\frac{1}{2}}\right)=(1 / 8)\left(s_{0, i+1}+6 s_{0, i}+s_{0, i-1}\right), \quad i=1(1) n-1 \text {. }
$$

Hence values $s_{0, i+\frac{1}{2}}, i=0(1) n-1$ (which are unknown ones on the boundary) are successively determined by coupling the above consistency relation and additional end conditions (1.4):

$$
\left\{\begin{array}{l}
\frac{1}{2}\left(s_{0, i+\frac{1}{2}}+s_{0, i-\frac{1}{2}}\right)=(1 / 8)\left(s_{0, i+1}+6 s_{0, i}+s_{0, i-1}\right), \quad i=1(1) n-1 \\
\nabla_{2}^{r} s_{0, n-\frac{1}{2}}=0
\end{array}\right.
$$

Values $s_{n, i+\frac{1}{2}}, s_{i+\frac{1}{2}, 0}$ and $s_{i+\frac{1}{2}, n}$ on the boundary are similarly determined by the consistency relation and end conditions (1.4). Values $s_{i, j+\frac{1}{2}}, i=1(1) n-1, j=0(1) n-1$ (which are unknown ones denoted by symbol * in Figure 1) are determined by

$$
\left\{\begin{array}{l}
(1 / 8)\left(\mathrm{s}_{i+1, j+\frac{1}{2}}+6 s_{i, j+\frac{1}{2}}+s_{i-1, j+\frac{1}{2}}\right) \\
\quad=\frac{1}{2}\left(s_{i+\frac{1}{2}, j+\frac{1}{2}}+s_{i-\frac{1}{2}, j+\frac{1}{2}}\right), i=1(1) n-1 \\
s_{0, j+\frac{1}{2}} \text { and } s_{n, j+\frac{1}{2}}(\text { which have been already determined). }
\end{array}\right.
$$

Similarly values $s_{i+\frac{1}{2}, j}, i=0(1) n-1, j=1(1) n-1$ are determined by

$$
\left\{\begin{array}{l}
(1 / 8)\left(s_{i+\frac{1}{2}, j+1}+6 s_{i+\frac{1}{2}, j}+s_{i+\frac{1}{2}, j-1}\right) \\
\quad=\frac{1}{2}\left(s_{i+\frac{1}{2}, j+\frac{1}{2}}+s_{i+\frac{1}{2}, j-\frac{1}{2}}\right), j=1(1) n-1 \\
s_{i+\frac{1}{2}, 0} \text { and } s_{i+\frac{1}{2}, n} \text { (which have been already determined). }
\end{array}\right.
$$

Hence we may have the following nine quantities in $\left[x_{i}, x_{i+1}\right] \times\left[y_{j}\right.$, $\left.y_{j+1}\right], i, j=0(1) n-1$ :

$$
s_{i, j}, s_{i, j+\frac{1}{2}}, s_{i, j+1} ; s_{i+\frac{1}{2}, j}, s_{i+\frac{1}{2}, j+\frac{1}{2}}, s_{i+\frac{1}{2}, j+1} ; s_{i+1, j}, s_{i+1, j+\frac{1}{2}}, s_{i+1, j+1} \text {. }
$$

In terms of these values $s$ may be easily represented at any points in $\left[x_{i}, x_{i+1}\right] \times\left[y_{j}, y_{j+1}\right]$ (see (4.15)).

Next we shall consider the so-called biquadratic histospline approximation determined by the conditions :

$$
\begin{gathered}
\int y_{j+1} \int_{y_{j-1}}^{x_{i+1}}[s(x, y)-f(x, y)] d x d y=0 \\
x_{i-1} \\
i, j=1(1) n-1
\end{gathered}
$$

with appropriate additional conditions. In this case we take the following $4 n$ conditions together with (1.9) : 


$$
\left\{\begin{array}{l}
\Delta_{1}^{r}\left(s_{0, j+1}+4 s_{0, j}+s_{0, j-1}\right) \\
\quad=\nabla_{1}^{r}\left(s_{n, j+1}+4 s_{n, j}+s_{n, j-1}\right)=0, j=1(1) n-1, \\
\Delta_{2}^{r}\left(s_{i+1,0}+4 s_{i, 0}+s_{i-1,0}\right) \\
\quad=\nabla_{2}^{r}\left(s_{i+1, n}+4 s_{i, n}+s_{i-1, n}\right)=0, i=1(1) n-1, \\
\left(\Delta_{1} \Delta_{2}\right)^{r} s_{0,0}=\left(\nabla_{1} \Delta_{2}\right)^{r} s_{n, 0}=\left(\Delta_{1} \nabla_{2}\right)^{r} s_{0, n}=\left(\nabla_{1} \nabla_{2}\right)^{r} s_{n, n} \\
\quad=0 .
\end{array}\right.
$$

Here we notice that the coefficient " 4 " in the above conditions is related to "4" in Lemma 3.1 which is the main tool in analyzing the histospline approximation. On using Lemmas 3.1-3.3, from (1.9) -(1.10) we have the block tridiagonal system of linear equations with $s_{i, j}, i, j=0(1) n$. Since $s$ depends upon $(n+2)^{2}$ parameters, we have to add four additional conditions to (1.10) toward the unique determination of $s$. Then we impose only one condition on each linear segment of the boundary, that is,

$$
\Delta_{1}^{r} s_{\frac{1}{2}, 0}=\Delta_{2}^{r} s_{n, \frac{1}{2}}=\nabla_{1}^{r} s_{n-\frac{1}{2}, n}=\nabla_{2}^{r} s_{0, n-\frac{1}{2}}=0 .
$$

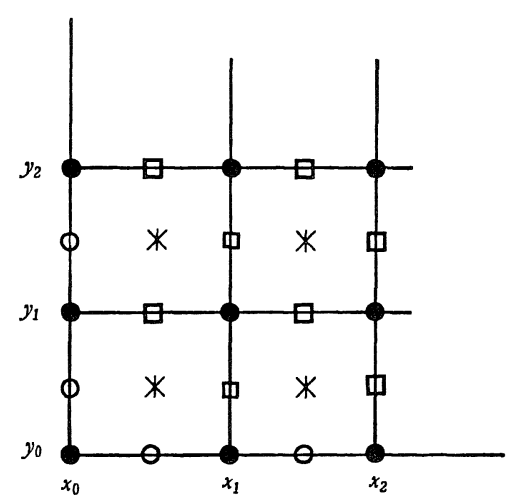

(i ) : determined by (1.9) and (1. 10)

(ii) 0 : determined by (i) and (1.11)

(iii) $\square$ : determined by (ii) and Lemma 3.6

(iv) $*$ : determined by (i), (ii), (iii) and Lemma 3.5

Figure 2

On referring Figure 2, similarly as in mid-point interpolation, $s$ may be uniquely determined.

\section{§2. Some Lemmas for Mid-Point Interpolation}

The following consistency relation is well known.

Lemma 2.1 ([2]). Let $s(x)=\sum_{i=-2}^{n-1} \alpha_{2} Q_{3}(x / h-i)$, then we have 


$$
\begin{aligned}
& (1 / 8)\left(s_{i+1}+6 s_{i}+s_{i-1}\right)=\frac{1}{2}\left(s_{i+\frac{1}{2}}+s_{i-\frac{1}{2}}\right) \\
& i=1(1) n-1 .
\end{aligned}
$$

On using this Lemma we have the following consistency relation for the biquadratic spline.

Lemma 2.2. Let $s(x, y)=\sum_{i, j=-2}^{n-1} \alpha_{i, j} Q_{3}(x / h-i) Q_{3}(y / h-j)$, then we have

$$
\begin{aligned}
& (1 / 64)\left\{s_{i+1, j+1}+s_{i+1, j-1}+s_{i-1, j+1}+s_{i-1, j-1}\right. \\
& \left.\quad+6\left(s_{i, j+1}+s_{i, j-1}+s_{i+1, j}+s_{i-1, j}\right)+36 s_{i, j}\right\} \\
& \quad=\frac{1}{4}\left(s_{i+\frac{1}{2}, j+\frac{1}{2}}+s_{i+\frac{1}{2}, j-\frac{1}{2}}+s_{i-\frac{1}{2}, j+\frac{1}{2}}+s_{i-\frac{1}{2}, j-\frac{1}{2}}\right) \\
& \quad i, j=1(1) n-1 .
\end{aligned}
$$

In order to transform the coefficient matrix of the system of linear equations with unknown $s_{i, j}$ into the block tridiagonal one, we shall require the following Lemmas 2.3 and 2.4. Let us denote the right hand side of equation (2.2) be $d_{i, j}, i, j=1(1) n-1$, then we have

Lemma 2.3. The boundary condition $\Delta_{1}^{r}\left(s_{0,0}+6 s_{0,1}+s_{0,2}\right)=0$ may be rewritten as follows:

$$
\begin{aligned}
s_{0,0} & +6 s_{0,1}+s_{0,2}+\sigma_{r}\left(s_{1,0}+6 s_{1,1}+s_{1,2}\right) \\
= & \sum_{i=1}^{r-1} \beta_{i}^{r} d_{i, 1}
\end{aligned}
$$

where we give some numerical values for the quantities $\sigma_{r}$ and $\beta_{i}^{r}$ for $r=$ 3, 4 :

Table 1

\begin{tabular}{l|l|lcl}
\hline$r$ & $\sigma_{r}$ & $\beta_{1}^{r}$ & $\beta_{z}^{r}$ & $\beta_{3}^{r}$ \\
\hline 3 & 7 & 72 & -8 & \\
4 & 6 & 65 & -10 & 1 \\
\hline
\end{tabular}

Lemma 2.4. The boundary condition $\left(\Delta_{1} \Delta_{2}\right)^{r} s_{0,0}=0$ may be rewritten as follows :

$$
s_{0,0}+\sigma_{r}\left(s_{0,1}+s_{1,0}\right)+\sigma_{r}^{2} s_{1,1}=\sum_{i, j=1}^{r-1} \beta_{i, j}^{r} d_{i, j},
$$

where we also give the numerical values for the quantities $\beta_{i, j}^{r}, i, j=1(1)$ $r-1$ for $r=3,4$ : 
Table 2.1

Values of $\beta_{t, j}^{3}$

\begin{tabular}{|c|c|c|}
\hline $\begin{array}{ll}j \\
i\end{array}$ & 1 & 2 \\
\hline 1 & 81 & -9 \\
\hline 2 & -9 & 1 \\
\hline
\end{tabular}

Table 2.2

Values of $64 \beta_{r, j}^{4}$

\begin{tabular}{|c|c|c|c|}
\hline$i_{i} \quad j$ & 1 & 2 & 3 \\
\hline 1 & 4225 & -650 & 65 \\
\hline 2 & -650 & 100 & -10 \\
\hline 3 & 65 & -10 & 1 \\
\hline
\end{tabular}

In order to prove the nonsingularity of the coefficient matrix obtained by using the above Lemmas and the boundedness of its inverse, we shall require the following

Lemma 2.5([6]). If $\sigma \neq 3+2 \sqrt{2}$, the tridiagonal $m \times m$ matrix $J_{1}(\sigma)$ is nonsingular and $\left\|J_{1}^{-1}(\sigma)\right\|$ is bounded for sufficiently large $m$, where $\|\cdot\|$ stands for the maximum norm and

$$
J_{1}(\sigma)=\left[\begin{array}{cccccc}
1 & \sigma & & & & \\
1 & 6 & 1 & & & \\
& \cdot & \cdot & \cdot & & \\
& & \cdot & \cdot & \cdot & \\
& & & 1 & 6 & 1 \\
& & & & \sigma & 1
\end{array}\right] .
$$

Let $I$ be the $m \times m$ unit matrix, the $m^{2} \times m^{2}$ coefficient matrix of the linear system with $s_{i, j}$ may be represented as follows :

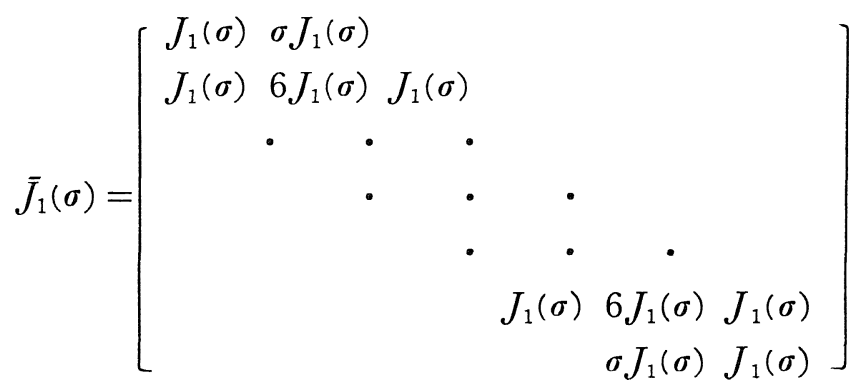




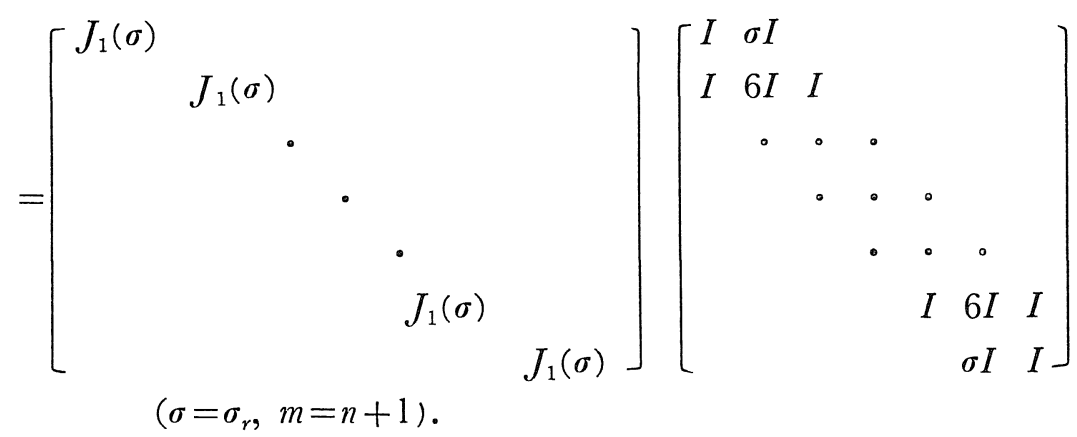

Hence on using the above auxiliary Lemma 2.5 we have the key lemma on the coefficient matrix of the system of linear equations with respect to $s_{i, j}, i, j=0(1) \mathrm{n}$.

Lemma 2.6. If $\sigma \neq 3+2 \sqrt{2}$, the above block tridiagonal matrix $\bar{J}_{1}(\sigma)$ is nonsingular and $\left\|\bar{J}_{1}^{-1}(\sigma)\right\|$ is bounded for sufficiently large $m$.

\section{§3. Some Lemmas for Histospline Approximation}

For histospline approximation we use the following consistency relation whose proof will be done by substituting products of $1, x$, $x^{2},\left(x-x_{i}\right)_{+}^{2}$ and $1, y, y^{2},\left(y-y_{j}\right)_{+}^{2}$.

Lemma 3.1. Let $s(x, y)=\sum_{i, j=-2}^{n-1} \alpha_{i, j} Q_{3}(x / h-i) Q_{3}(y / h-j)$, then we have

$$
\begin{aligned}
& \text { (1/36) }\left\{s_{i+1, j+1}+s_{i+1, j-1}+s_{i-1, j+1}+s_{i-1, j-1}\right. \\
& \left.\quad+4\left(s_{i+1, j}+s_{i-1, j}+s_{i, j+1}+s_{i, j-1}\right)+16 s_{i, j}\right\} \\
& \quad=\frac{1}{4} h^{-2} \int_{y_{j-1}}^{y_{j+1}} \int_{x_{i-1}}^{x_{i+1}} s(x, y) d x d y, i, j=1(1) n-1 .
\end{aligned}
$$

Let the right hand side of the above equation be $\bar{d}_{i, j}$, then we have

Lemma 3.2. The boundary condition $\Delta_{1}^{r}\left(s_{0,0}+4 s_{0,1}+s_{0,2}\right)=0$ may be rewritten as follows:

$$
\begin{aligned}
s_{0,0} & +4 s_{0,1}+s_{0,2}+\bar{\sigma}_{r}\left(s_{1,0}+4 s_{1,1}+s_{1,2}\right) \\
= & \sum_{i=1}^{r-1} \bar{\beta}_{i}^{r} \bar{d}_{i, 1}
\end{aligned}
$$

where we give some numerical values for the quantities $\overline{\boldsymbol{\sigma}}_{r}$ and $\bar{\beta}_{i}^{r}$ : 
Table 3

\begin{tabular}{c|c|ccc}
\hline$r$ & $\bar{\sigma}_{r}$ & $\bar{\beta}_{1}^{r}$ & $\bar{\beta}_{2}^{r}$ & $\bar{\beta}_{3}^{r}$ \\
\hline 3 & 5 & 42 & -6 & \\
4 & 4 & 37 & -8 & 1 \\
\hline
\end{tabular}

Lemma 3.3. The boundary condition $\left(\Delta_{1} \Delta_{2}\right)^{r} s_{0,0}=0$ may be rewritten as follows:

$$
s_{0,0}+\bar{\sigma}_{r}\left(s_{0,1}+s_{1,0}\right)+\bar{\sigma}_{r}^{2} s_{1,1}=\sum_{i, j=1}^{r-1} \bar{\beta}_{i, j}^{r} \bar{d}_{i, j}
$$

where values for the quantities $\bar{\beta}_{i, j}$ are as follows:

Table 4.1

Values of $\bar{\beta}_{s, j}^{3}$

\begin{tabular}{|c|c|c|}
\hline$j$ & 1 & 2 \\
\hline 1 & 49 & -7 \\
\hline 2 & -7 & 1 \\
\hline
\end{tabular}

Table 4.2

Values of $36 \bar{\beta}_{1, j}^{4}$

\begin{tabular}{rr|rrr}
\hline$i$ & & $j$ & \multicolumn{1}{c}{2} & \multicolumn{1}{c}{3} \\
\hline 1 & 1 & 1369 & -296 & 37 \\
2 & -296 & 64 & -8 \\
3 & 37 & -8 & 1 \\
\hline
\end{tabular}

Let us define an $m \times m$ tridiagonal matrix $J_{2}(\sigma)$ by

$$
J_{2}(\sigma)=\left[\begin{array}{ccccccc}
1 & \sigma & & & & & \\
1 & 4 & 1 & & & & \\
& \bullet & \bullet & \bullet & & & \\
& & \cdot & \bullet & \cdot & & \\
& & & \cdot & \cdot & \cdot & \\
& & & & 1 & 4 & 1 \\
& & & & & \sigma & 1
\end{array}\right]
$$

then the $m^{2} \times m^{2}$ coefficient matrix of the system of linear equations with $s_{i, j}$ is given by 


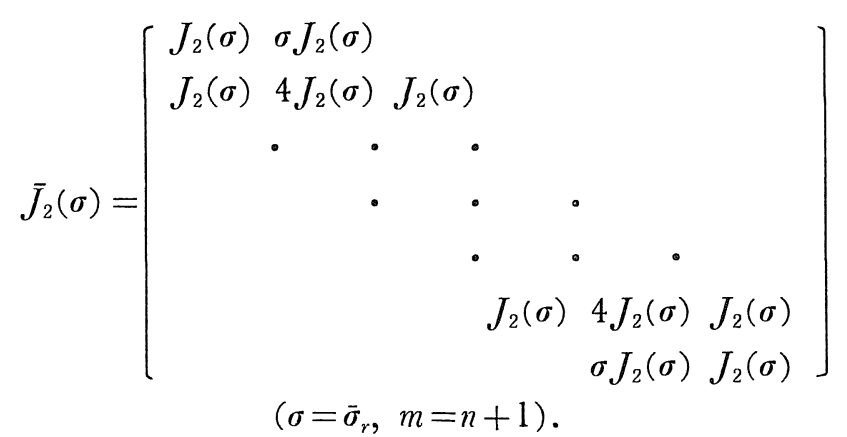

In this case we have

Lemma 3.4. If $\sigma \neq 2+\sqrt{3}$, the above block tridiagonal matrix $\bar{J}_{2}(\sigma)$ is nonsingular and $\left\|\bar{J}_{2}^{-1}(\sigma)\right\|$ is bounded for sufficientlv large $m$.

In order to determine values $s_{i+\frac{1}{2}, j}, s_{i, j+\frac{1}{2}}$ and $s_{i+\frac{1}{2}, j+\frac{1}{2}}$, the following consistency relations are required.

Lemma 3.5. On each subinterval $\left[x_{i}, x_{i+1}\right] \times\left[y_{j}, y_{j+1}\right]$, we have

$$
\begin{aligned}
& \int_{y_{j}}^{y_{j+1}} \int_{x_{i}}^{x_{i+1}} s(x, y) d x d y=\left(h^{2} / 36\right)\left\{s_{i, j}+s_{i, j+1}+s_{i+1, j}+s_{i+1, j+1}\right. \\
& \left.\quad+4\left(s_{i, j+\frac{1}{2}}+s_{i+\frac{1}{2}, j+1}+s_{i+\frac{1}{2}, j}+s_{i+1, j+\frac{1}{2}}\right)+16 s_{i+\frac{1}{2}, j+\frac{1}{2}}\right\}
\end{aligned}
$$

Proof. Since $s$ is an algebraic polynomial of degree 2 or less with respect to $x$ and $y$ in subregion $\left[x_{i}, x_{i-1}\right] \times\left[y_{j}, y_{j+1}\right]$, the above relation is easily proved.

On combining Lemmas 2.1 and 3.5, we have

Lemma 3.6. On the interval $\left[x_{i}, x_{i+1}\right] \times\left[y_{j-1}, y_{j+1}\right]$, we have

$$
\begin{aligned}
& s_{i+\frac{1}{2}, j+1}+4 s_{i+\frac{1}{2}, j}+s_{i+\frac{1}{2}, j-1} \\
& \quad=(9 / 2) h^{-2} \int_{y_{j-1}}^{y_{j+1}} \int_{x_{i}}^{x_{i+1}} s(x, y) d x d y \\
& \quad-\frac{1}{4}\left\{\left(s_{i+1, j+1}+4 s_{i+1, j}+s_{i+1, j-1}\right)\right. \\
& \left.\quad+\left(s_{i, j+1}+4 s_{i, j}+s_{i, j-1}\right)\right\} .
\end{aligned}
$$




\section{§ 4. Main Results for Mid-Point Interpolation}

In what follows, for any finite dimensional row vector $\left(c_{i}\right)$, let us denote the transposed one (i.e., column vector) by $\left(c_{i}\right)^{\prime}$.

On combining Lemmas 2.2-2.4 we have

$$
(1 / 64) \bar{J}_{1}\left(\sigma_{r}\right)\left(s_{0}, s_{1}, \ldots, s_{n}\right)^{\prime}=\left(d_{0}, d_{1}, \ldots, d_{n}\right)^{\prime}
$$

where

$$
\begin{aligned}
& s_{j}=\left(s_{0, j}, s_{1, j}, \ldots, s_{n, j}\right)^{\prime} \\
& d_{j}=\left(d_{0, j}, d_{1, j}, \ldots, d_{n, j}\right)^{\prime} .
\end{aligned}
$$

By use of interpolation condition (1.2), the numbers $d_{i, j}, i, j=1(1)$ $n-1$ are given by

$$
\begin{aligned}
d_{i, j} & =\frac{1}{4}\left(f_{i+\frac{1}{2}, j+\frac{1}{2}}+f_{i+\frac{1}{2}, j-\frac{1}{2}}+f_{i-\frac{1}{2}, j+\frac{1}{2}}\right. \\
& \left.+f_{i-\frac{1}{2}, j-\frac{1}{2}}\right) .
\end{aligned}
$$

The remaining terms $d_{0, j}, d_{n, j}, d_{i, 0}$ and $d_{i, n}, i, j=0(1) n$ may be represented in terms of the above $d_{i, j}$ :

$$
\left\{\begin{array}{l}
d_{0, j}=(1 / 64) \sum_{i=1}^{r-1} \beta_{i}^{r} d_{i, j}, \quad d_{n, j}=(1 / 64) \sum_{i=1}^{r-1} \beta_{i}^{r} d_{n-i, j} \\
d_{i, 0}=(1 / 64) \sum_{j=1}^{r-1} \beta_{j}^{r} d_{i, j}, \quad d_{i, n}=(1 / 64) \sum_{j=1}^{r-1} \beta_{j}^{r} d_{i, n-j} \\
d_{0,0}=(1 / 64) \sum_{i, j=1}^{r-1} \beta_{i, j}^{r} d_{i, j} \\
d_{0, n}=(1 / 64) \sum_{i, j=1}^{r-1} \beta_{, j}^{r} d_{i, n-j} \\
d_{n, 0}=(1 / 64) \sum_{i, j=1}^{r-1} \beta_{i, j}^{r} d_{n-i, j} \\
d_{n, n}=(1 / 64)_{i, j=1}^{r-1} \beta_{i, j}^{r} d_{n-i, n-j} .
\end{array}\right.
$$

On using Lemma 2.6, the system of linear equations with $s_{i, j}, i, j=$ $0(1) n$ may be solved for sufficiently large $n$. Before we proceed with analysis, we notice that it is very easy to solve the system (4.1). By multiplying the block diagonal matrix whose diagonal elements are $J_{1}^{-1}\left(\sigma_{r}\right)$ on the both side of (4.1), we have 


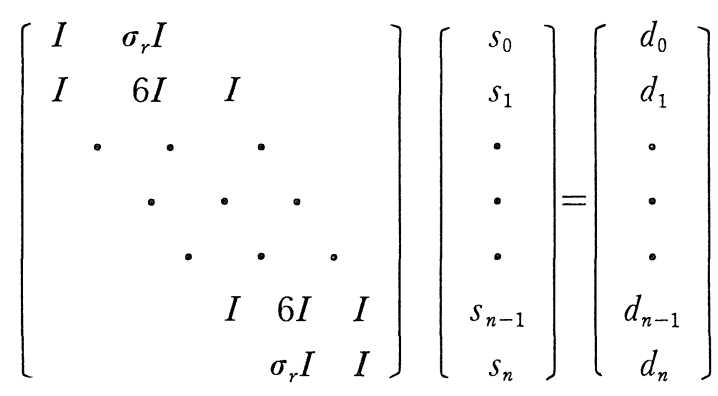

where let us denote $J_{1}^{-1}\left(\sigma_{r}\right) d_{i}$ by the same symbol $d_{i}, i=0(1) n$. From (4.5), we have

$$
\begin{gathered}
\left(s_{i, 0}, s_{i, 1}, \ldots, s_{i, n}\right)^{\prime}=J_{1}^{-1}\left(\sigma_{r}\right)\left(d_{i, 0}, d_{i, 1}, \ldots, d_{i, n}\right)^{\prime} \\
i=0(1) n .
\end{gathered}
$$

That is, $s_{i, j}, i, j=0(1) n$ may be calculated by solving $2(n+1)$ systems of linear equations which are different only in constant terms. For any given vector $k=\left(k_{0}, k_{1}, \ldots, k_{n}\right)$, we only have to solve the matrix problem

$$
\left[\begin{array}{cccccccc}
1 & \sigma_{r} & & & & & & \\
1 & 6 & 1 & & & & & \\
& \cdot & \bullet & \cdot & & & & \\
& & \bullet & \cdot & \cdot & & & \\
& & & \cdot & \cdot & \cdot & & \\
& & & & \cdot & \cdot & \cdot & \\
& & & & & 1 & 6 & 1 \\
& & & & & & \sigma_{r} & 1
\end{array}\right]\left(\begin{array}{c}
z_{0} \\
z_{1} \\
\cdot \\
\cdot \\
\cdot \\
\cdot \\
z_{n-1} \\
z_{n}
\end{array}\right]=\left(\begin{array}{c}
k_{0} \\
k_{1} \\
\cdot \\
\cdot \\
\cdot \\
\cdot \\
k_{n-1} \\
k_{n}
\end{array}\right) .
$$

This problem can be directly solved by the following simple algorithm :

$$
\left\{\begin{array}{l}
w_{0}=\sigma_{r}, \quad w_{i}=1 /\left(6-w_{i-1}\right), \quad i=1(1) n-1 \\
g_{0}=k_{0}, \quad g_{i}=\left(k_{\imath}-w_{i-1}\right) /\left(6-w_{i-1}\right), \quad i=1(1) n \\
z_{n}=g_{n}, \quad z_{i}=g_{\imath}-w_{i} z_{i+1}, \quad i=0(1) n-1 .
\end{array}\right.
$$

Since $w_{0}\left(=\sigma_{r}\right)=6$ for $r=4$, it is impossible to calculate $w_{1}$. But in this case, from the first and second equations of (4.7) we have

$$
\begin{aligned}
& z_{0}+6 z_{1}=k_{0} \\
& z_{0}+6 z_{1}+z_{2}=k_{1}
\end{aligned}
$$


from which follows

$$
z_{2}=k_{1}-k_{0} \text {. }
$$

Hence we have a system of linear equations which is directly solved by the above algorithm :

$$
\begin{aligned}
& {\left[\begin{array}{cccccccc}
1 & 0 & & & & & & \\
1 & 6 & 1 & & & & & \\
& \cdot & \cdot & \cdot & & & & \\
& & \cdot & \cdot & \cdot & & & \\
& & & \cdot & \cdot & \cdot & & \\
& & & & \cdot & \cdot & \cdot & \\
& & & & & 1 & 6 & 1 \\
& & & & & & 6 & 1
\end{array}\right]\left(\begin{array}{c}
z_{2} \\
z_{3} \\
\cdot \\
\cdot \\
\cdot \\
\cdot \\
z_{n-1} \\
z_{n}
\end{array}\right)=\left(\begin{array}{c}
k_{1}-k_{0} \\
k_{3} \\
\cdot \\
\cdot \\
\cdot \\
\cdot \\
k_{n-1} \\
k_{n}
\end{array}\right)} \\
& z_{1}=k_{2}-6 z_{2}-z_{3}, \quad z_{0}=k_{0}-6 z_{1} .
\end{aligned}
$$

Let $k$ and $p$ be integers such that $0 \leq k \leq p$ and $4 \leq p$. Then, for $f \in C^{p}(\Omega)$, let us denote

$$
\begin{aligned}
& f^{(k x)}(x, y)=\frac{\partial^{k} f}{\partial x^{k}}, \quad f^{(k y)}(x, y)=\frac{\partial^{k} f}{\partial y^{k}} \\
& g(x, y)=f^{(4 x)}(x, y)+f^{(4 y)}(x, y) \\
& e(x, y)=f(x, y)-s(x, y) .
\end{aligned}
$$

By the means of Taylor series expansion, we have

$$
\left\{\begin{array}{c}
(1 / 64)\left\{e_{i+1, j+1}+e_{i+1, j-1}+e_{i-1, j+1}+e_{i-1, j-1}\right. \\
\left.\quad+6\left(e_{i, j+1}+e_{i, j-1}+e_{i+1, j}+e_{i-1, j}\right)+36 e_{i, j}\right\} \\
\quad=\left(h^{4} / 128\right) g_{i, j}+O\left(h^{6}\right), \quad i, j=1(1) n-1, \\
\Delta_{1}^{r}\left(e_{0, j+1}+6 e_{0 j}+e_{0, j-1}\right)=O\left(h^{r}\right), \cdots \\
\left(\Delta_{1} \Delta_{2}\right)^{r} s_{0,0}=O\left(h^{r}\right), \cdots
\end{array}\right.
$$

Hence, on using Lemmas 2.3, 2.4 and 2.6, we have

Theorem 1. If $f(x, y) \in C^{6}(\Omega)$, we have

$$
\begin{aligned}
\text { (i ) } & f_{i, j}-s_{i, j}=\left(h^{4} / 128\right) g_{i, j}+O\left(h^{\min (r, 6)}\right), \quad h \rightarrow 0 \\
& i, j=0(1) n ; \\
\text { (ii) } & f_{i, j}-s_{i, j}=\left(h^{4} / 128\right) g_{i, j}+O\left(h^{6}\right), \quad h \rightarrow 0
\end{aligned}
$$

for any mesh points $\left(x_{i}, y_{j}\right)$ bounded away from the boundary.

Proof of (ii). From (4.1), we have 


$$
\bar{J}_{1}\left(\sigma_{r}\right)\left(e_{0}, e_{1}, \ldots, e_{n}\right)^{\prime}=\left(d_{0}, d_{1}, \ldots, d_{n}\right)^{\prime}
$$

where

$$
\left\{\begin{array}{l}
e_{j}=\left(e_{0, j}, e_{1, j}, \ldots, e_{n, j}\right)^{\prime} \\
d_{j}=\left(d_{0, j}, d_{1, j}, \ldots, d_{n, j}\right)^{\prime} .
\end{array}\right.
$$

Since $d_{i, j}=\left(8^{2} h^{4} / 128\right) g_{i, j}+\cdots, i, j=1(1) n-1$, on using the well known technique in (Kershaw [3]) we have

$$
\text { "the } i \text {-th component of the vector } J_{1}^{-1}\left(\sigma_{r}\right) d_{j} \text { " }
$$

$$
=\left(8 h^{4} / 128\right) g_{i, j}+\cdots
$$

for any mesh points $\left(x_{i}, y_{j}\right)$ bounded away from the boundary. In a manner similar to the derivation of (4.7), we have

$$
\begin{aligned}
& \left(e_{i, 0}, e_{i, 1}, \ldots, e_{i, n}\right)^{\prime} \\
& \quad=J_{1}^{-1}\left(\sigma_{r}\right)\left(\left(J_{1}^{-1}\left(\sigma_{r}\right) d_{0}\right)_{i},\left(J_{1}^{-1}\left(\sigma_{r}\right) d_{1}\right)_{\imath}, \ldots,\left(J_{1}^{-1}\left(\sigma_{r}\right) d_{n}\right)_{i}\right)^{\prime},
\end{aligned}
$$

where for any vector $c=\left(c_{0}, c_{1}, \ldots, c_{n}\right)^{\prime}$, let us denote its $i$-th component $c_{i}$ by $\left(c_{0}, c_{1}, \ldots, c_{n}\right)_{i}^{\prime}$.

On using again Kershaw's technique we have the desired result.

Next we shall consider the global error estimation. By using Theorem 1, (1.7) and (1.8), we have

$$
\left\{\begin{array}{l}
e_{i, j+\frac{1}{2}}=\left(h^{4} / 128\right) f_{i, j+\frac{1}{2}}^{(3 x)}+\cdots \\
e_{i+\frac{1}{2}, j}=\left(h^{4} / 128\right) f_{i+\frac{1}{2}, j}^{(3 y)}+\cdots
\end{array}\right.
$$

for any points $\left(x_{i}, y_{j+\frac{1}{2}}\right)$ and $\left(x_{i+\frac{1}{2}}, y_{j}\right)$ bounded away from the boundary. Since $s$ is a quadratic polynomial with respect to $x$ and $y$,

$$
\begin{aligned}
& s(x, y)=s_{i, j} p\left\{\left(x-x_{i}\right) / h,\left(y-y_{j}\right) / h\right\} \\
& \quad+s_{i, j+1} p\left\{\left(x-x_{i}\right) / h,\left(y_{j+1}-y\right) / h\right\}+\cdots \\
& \quad+s_{i, j+\frac{1}{2}} q\left\{\left(x-x_{i}\right) / h,\left(y-y_{j+\frac{1}{2}}\right) / h\right\}+\cdots \\
& \quad+s_{i+\frac{1}{2}, j+\frac{1}{2}} r\left\{\left(x-x_{i+\frac{1}{2}}\right) / h,\left(y-y_{j+\frac{1}{2}}\right) / h\right\}
\end{aligned}
$$

where

$$
\left\{\begin{array}{l}
p(x, y)=4\left(x-\frac{1}{2}\right)(x-1)\left(y-\frac{1}{2}\right)(y-1) \\
q(x, y)=-8\left(x-\frac{1}{2}\right)(x-1)\left(y-\frac{1}{2}\right)\left(y+\frac{1}{2}\right) \\
r(x, y)=1-4 x^{2}-4 y^{2}+16 x^{2} y^{2} .
\end{array}\right.
$$

Hence we have

Theorem 2. For any points bounded away from the boundary, we have 


$$
\begin{aligned}
& f(x, y)-s(x, y)=-\left(h^{3} / 6\right)\left[\left\{\frac{1}{4}-\left(x-\frac{1}{2}\right)^{2}\right\} \times\right. \\
& \left.\left(x-\frac{1}{2}\right) f_{i+\frac{1}{2}, j+\frac{1}{2}}^{(3 x)}+\left\{\frac{1}{4}-\left(y-\frac{1}{2}\right)^{2}\right\}\left(y-\frac{1}{2}\right) f_{i+\frac{1}{2}, j+\frac{1}{2}}^{(3 y)}\right] \\
& \quad+O\left(h^{4}\right), h \rightarrow 0, \quad x_{i} \leq x \leq x_{i+1}, \quad y_{j} \leq y \leq y_{j+1} .
\end{aligned}
$$

\section{§5. Histospline Approximation}

On using Lemmas 3.1-3.3 we have a system of linear equations with $s_{i, j}$ whose coefficient matrix is block tridiagonal. From Lemma 3.4, $s_{i, j}, i j=0(1) n$ are uniquely determined for sufficiently large $n$. Next we shall consider the asymptotic error estimation :

Theorem 3. If $f(x, y) \in C^{6}(\Omega)$, we have

$$
\begin{array}{ll}
\text { (i ) } & f_{i, j}-s_{i, j}=\left(h^{4} / 180\right) g_{i, j}+O\left(h^{\min (r, 6)}\right), h \rightarrow 0 \\
& i, j=0(1) n ; \\
\text { (ii ) } & f_{i, j}-s_{i, j}=\left(h^{4} / 180\right) g_{i, j}+O\left(h^{6}\right), h \rightarrow 0
\end{array}
$$

for any mesh points $\left(x_{i}, y_{j}\right)$ bounded away from the boundary.

Proof. From Lemma 3.1, on expanding $f$ about $\left(x_{i}, y_{j}\right)$ we have

$$
\left\{\begin{array}{l}
(1 / 36)\left\{e_{i+1, j+1}+e_{i+1, j-1}+e_{i-1, j+1}+e_{i-1, j-1}\right. \\
\left.\quad+4\left(e_{i, j+1}+e_{i, j-1}+e_{i+1, j}+e_{i-1, j}\right)+16 e_{i, j}\right\} \\
\quad=\left(h^{4} / 180\right) g_{i, j}+O\left(h^{m i n(r, 6)}\right), \\
\Delta_{1}^{r}\left(e_{0, j+1}+4 e_{0, j}+e_{0, j-1}\right)=O\left(h^{r}\right), \cdots \\
\left(\Delta_{1} \Delta_{2}\right)^{r} e_{0,0}=O\left(h^{r}\right), \cdots .
\end{array}\right.
$$

Application of Lemmas $3.2-3.4$ to (5.2) yields asymptotic expansion (i). The proof of (ii) is similar to that of (ii) in Theorem 1. By use of Theorem 3 and Lemma 3.6, we also have

$$
\begin{aligned}
& f_{i+\frac{1}{2}, j+\frac{1}{2}}-s_{i+\frac{1}{2}, j+\frac{1}{2}}=-\left(13 h^{4} / 5760\right) g_{i+\frac{1}{2}, j+\frac{1}{2}} \\
& \quad+O\left(h^{6}\right), h \rightarrow 0
\end{aligned}
$$

for any mid-points $\left(x_{i+\frac{1}{2}}, y_{j+\frac{1}{2}}\right)$ bounded away from the boundary.

\section{§ 6. Numerical Illustration}

In this section we shall consider the application of the above stated methods by taking $f(x, y)=\exp (x+y)$. Here we have given 
the numerical results only for $r=4$. The approximate rate of decrease of the errors, $O\left(h^{\alpha}\right)$, where $\alpha$ is computed from the observed decrease in the error from $h=1 / 16$ to $1 / 32$, is given in parentheses. An comparison with Theorems 1 and 3 shows excellent agreement with the asymptotic rates.

\section{Table 5}

Observed errors of biquadratic spline interpolation at mid-points.

\begin{tabular}{c|cccc}
\hline$(x, y)$ & $h$ & $1 / 16$ & $1 / 32$ & \\
\hline$(0,0)$ & $0.337-3^{*}$ & $0.186-4$ & $0.109-5$ & $(4.1)$ \\
$\left(0, \frac{1}{2}\right)$ & $0.281-3$ & $0.155-4$ & $0.913-6$ & $(4.1)$ \\
$(0,1)$ & $0.738-3$ & $0.451-4$ & $0.280-5$ & $(4.0)$ \\
$\left(\frac{1}{2}, \frac{1}{2}\right)$ & $0.116-4$ & $0.648-6$ & $0.405-7$ & $(3.9)$ \\
$\left(\frac{1}{2}, 1\right)$ & $0.471-3$ & $0.331-4$ & $0.220-5$ & $(3.9)$ \\
$(1,1)$ & $0.152-2$ & $0.107-3$ & $0.714-5$ & $(3.9)$ \\
\hline
\end{tabular}

* We denote $0.337 \times 10^{-3}$ by $0.337-3$.

Table 6

Observed errors of biquadratic histospline approximation.

\begin{tabular}{c|cccc}
\hline$(x, y)$ & $h$ & $1 / 16$ & $1 / 32$ & \\
\hline$(0,0)$ & $1 / 8$ & $0.136-4$ & $0.796-6$ & $(4.1)$ \\
$\left(0, \frac{1}{2}\right)$ & $0.205-3$ & $0.113-4$ & $0.665-6$ & $(4.1)$ \\
$(0,1)$ & $0.535-3$ & $0.328-4$ & $0.204-5$ & $(4.0)$ \\
$\left(\frac{1}{2}, \frac{1}{2}\right)$ & $0.128-4$ & $0.462-6$ & $0.288-7$ & $(4.0)$ \\
$\left(\frac{1}{2}, 1\right)$ & $0.346-3$ & $0.241-4$ & $0.160-5$ & $(4.0)$ \\
$(1,1)$ & $0.111-2$ & $0.738-4$ & $0.520-5$ & $(3.9)$ \\
\hline
\end{tabular}

The observed errors $e_{M}$ and $e_{H}$ at $\left(\frac{1}{2}, \frac{1}{2}\right)$ associated with midpoint interpolation and histospline are $0.648-6,0.405-7$ and $0.462-6$, $0.288-7$, respectively, for $h=1 / 16$ and $1 / 32$. Hence we have

$e_{M} / e_{H}=1.403 \cdots, 1.406 \cdots$ for $h=1 / 16$ and $1 / 32$. On the other hand, the theoretical value for $e_{M} / e_{H}$ is $180 / 128=1.406 \cdots$.

From Tables 5 and 6 we also have at $\left(\frac{1}{2}, \frac{1}{2}\right)$

$$
(1 / 52)\left(180 e_{M}-128 e_{H}\right) \fallingdotseq-3.5 \times 10^{-12} \text { for } h=1 / 32 \text {. }
$$


This result is predicted by the following relation (which is easily obtained from Theorems 1 and 3):

$$
(1 / 52)\left(180 e_{M}-128 e_{H}\right)=O\left(h^{6}\right)
$$

for any mid-points $\left(x_{i+1 / 2}, y_{j+1 / 2}\right)$ bounded away from the boundary.

\section{References}

[1] Ahlberg, J., Nilson, E. and Walsh, J., The theory of splines and their applications, Academic Press, New York, 1967.

[2] Kammerer, W., Reddien, G. and Varga, R., Quadratic splines, Numer. Math., 22 (1974), 241-259.

[3] Kershaw, D., The orders of approximation of the first derivatives of cubic splines at the knots, Math. Comp., 26 (1972), 191-198.

[4] Lucas, T., Error bounds for interpolating cubic splines under various end conditions, SIAM J. Numer. Anal., 11 (1974), 569-584.

[5] Neuman, E., Quadratic splines and histospline projection, J. Approximation Theory, 29 (1980), 297-304.

[6] Usmani, R. and Sakai, M., A note on quadratic interpolation at mid-points. BIT 22 (1982), 261-267. 